\title{
Clinical and morphological characteristics in Streptococcus bovis endocarditis: a comparison with other causative microorganisms in 177 cases
}

\author{
I Kupferwasser, H Darius, A M Müller, S Mohr-Kahaly, T Westermeier, H Oelert, \\ R Erbel, J Meyer
}

\begin{abstract}
Aim-To compare the clinical and morphological characteristics of patients with Streptococcus bovis endocarditis with those of patients with endocarditis caused by other microorganisms.

Methods-177 consecutive patients (Streptococcus bovis, 22; other streptococci, 94; staphylococci, 44; other, 17) with definite infective endocarditis according to the Duke criteria were included. All patients underwent transthoracic and transoesophageal echocardiography. In 88 patients, findings from surgery/necropsy were obtained.

Results $-S$ bovis endocarditis was associated with older patients, with a higher mortality $(p=0.04)$, and with a higher rate of cardiac surgery $(p<0.001)$ than other microorganisms, although embolic events were observed less often $(p=0.02)$. Pathological gastrointestinal lesions were detected in $45 \%$ of the patients. Multiple valves were affected in $68 \%$ of the patients with $S$ bovis endocarditis and in $20 \%$ of those with other organisms $(p<0.001)$. Moderate or severe regurgitation occurred more often in $S$ bovis endocarditis than with other microorganisms $(p=0.05)$. When surgery or necropsy was performed, infectious myocardial infiltration of the left ventricle was confirmed histopathologically in $36 \%$ of the patients with $S$ bovis endocarditis and in $10 \%$ of those with other organisms $(p=0.002)$. Conclusions-S bovis endocarditis is a severe illness because of the more common involvement of multiple valves, and of the frequent occurrence of haemodynamically relevant valvar regurgitation and infectious myocardial infiltration. (Heart 1998;80:276-280)
\end{abstract}

Keywords: infective endocarditis; Streptococcus bovis transoesophageal echocardiography; valvar disease

Elderly people appear to form an increasing proportion of patients with infective endocarditis. ${ }^{12}$ Because they seem to be particularly prone to Streptococcus bovis endocarditis, it is important to evaluate the characteristics of the disease. ${ }^{34}$ Since the end of the 1970s several studies have reported an association between pathological gastrointestinal lesions and $S$ bovis endocarditis. ${ }^{5-10}$ A few anecdotal reports have also described an unusual morphological pattern of the disease. ${ }^{11}{ }^{12} \mathrm{Up}$ to now the clinical and morphological features have been less clearly defined.

Recent echocardiographic imaging techniques have improved the morphological evaluation in patients with infective endocarditis to a major extent. Thus transoesophageal echocardiography is now accepted as the imaging method of choice in infective endocarditis. ${ }^{13-18}$ Case control studies of the clinical and morphological features of infective endocarditis in relation to the causative microorganisms in a large group of patients using transoesophageal echocardiography are, however, scarce. We therefore undertook this study to evaluate the clinical and morphological characteristics of endocarditis caused by $S$ bovis in comparison with other causative microorganisms in a large patient group with definite infective endocarditis.

\section{Methods}

PATIENTS

Between March 1983 and July 1996, 638 consecutive patients with suspected infective endocarditis were referred to our echocardiographic laboratory. In 265 patients definite infective endocarditis according to the Duke criteria ${ }^{19}$ could be confirmed. Eighty eight patients with prosthetic valve endocarditis, fungal, or culture negative endocarditis were excluded from the study. The remaining 177 patients formed the present study group. All patients had undergone transthoracic and transoesophageal echocardiographic investigations during the acute phase of infective endocarditis. There were 104 men and 73 women, mean (SD) age 57 (16) years (range 21 to 76 ). Morphological findings were obtained in 70 patients who had heart surgery and in 18 patients from necropsy; in these patients the diagnosis of infective endocarditis was confirmed by the pathological findings. In 89 patients the diagnosis was made on the basis of clinical criteria (two major criteria, $\mathrm{n}=75$; one major and three minor criteria, $\mathrm{n}=14$ ). Twenty one patients with $S$ bovis endocarditis underwent gastroscopy and colonoscopy. Patients were followed regularly at the outpatient clinic at 12 monthly intervals until death. If a patient missed a follow up investigation, both the patient and his general practitioner were contacted by telephone or a medical questionnaire. Mean (SD) follow up time was 50 (41) months. 
Table 1 Personal data, clinical characteristics, and pre-existing conditions in 177 patients with endocarditis

\begin{tabular}{|c|c|c|c|c|c|}
\hline & $S$ bovis & Other strep ${ }^{*}$ & Staph $\uparrow$ & Other $\neq$ & $p$ value \\
\hline Number & $22(12.4 \%)$ & $94(53.1 \%)$ & $44(24.9 \%)$ & $17(9.6 \%)$ & ND \\
\hline Mean age (years) (range) & 67 (49 to 76$)$ & $57(29$ to 74$)$ & $53(21$ to 72$)$ & $54(32$ to 62$)$ & $<0.001$ \\
\hline $\operatorname{Sex}(F / M)(\%)$ & $46 / 54$ & $39 / 61$ & $41 / 59$ & $47 / 53$ & NS \\
\hline Surgical treatment $(\%)$ & 73 & 34 & 34 & 41 & $<0.001$ \\
\hline Necropsy $(\%)$ & 18 & 9 & 11 & 6 & ND \\
\hline Previous endocarditis (\%) & 5 & 9 & 5 & 0 & NS \\
\hline Intravenous drug abuser (\%) & 0 & 1.1 & 2.3 & 0 & NS \\
\hline Pre-existing cardiac disease $(\%)$ & 18 & 30 & 27 & 24 & NS \\
\hline Extracardiac disease $(\%)$ & 32 & 20 & 11 & 12 & NS \\
\hline Therapeutic delay (days) (SD) & $14(6)$ & $9(4)$ & $7(4)$ & $9(7)$ & $<0.001$ \\
\hline
\end{tabular}

$N D$, not done; $p$ value, $S$ bovis $v$ non-S bovis endocarditis; therapeutic delay, time between the onset of endocarditis related symptoms and appropriate antibiotic treatment.

${ }^{\star}$ Streptococci other than $S$ bovis, including $58 S$ viridans, $16 S$ pyogenes, 13 enterococci, 4 pneumococci, $3 S$ intermedius.

†Staphylococci including $32 S$ aureus, $12 S$ epidermidis.

‡Rare microorganisms including 1 serratia, $2 \mathrm{H}$ influenzae, $1 \mathrm{H}$ parainfluenzae, 2 proteus, 2 pseudomonas, 1 klebsiella, $2 \mathrm{E}$ coli, 6 mixed Gram positive cultures.

MICROBIOLOGY

Blood cultures were positive in 169 patients $(95.5 \%)$. Cultures from valve tissue were positive in another eight $(4.5 \%)$. Staphylococci or streptococci accounted for the majority of cases. In 17 patients $(9.6 \%)$ rare organisms were isolated as the probable cause of infective endocarditis. Patients were divided into four groups according to the microorganisms isolated (table 1).

\section{ECHOCARDIOGRAPHY}

Transthoracic and transoesophageal echocardiographic examinations were performed with commercially available ultrasound units. For transthoracic studies, $2.5 \mathrm{MHz}$ transducers were used. A $3.75 \mathrm{MHz}$ or $5 \mathrm{MHz}$ phased array transducer or mechanical sector scanner was used for monoplane, biplane, and multiplane transoesophageal studies. The transoesophageal examinations were performed in the left lateral decubitus position after obtaining informed consent and after a six hour fasting period. Before the probe was introduced into the oesophagus a local pharyngeal anaesthetic was given. Sedation was routinely achieved with diazepam (2.5 to $10 \mathrm{mg}$ intravenously). Antibiotic prophylaxis (amoxicillin $1.5 \mathrm{mg}$ ) was given one hour before and four hours after the procedure. All investigations were carried out without any complications.

\section{Echocardiographic definition of vegetation}

A valvar vegetation was defined as an additional oscillating or fixed mass adherent to a leaflet, distinct in echogenic structure, and having motion independent of the remainder of the leaflet. It had to be detectable throughout the complete cardiac cycle and apparent in multiple views. Diffuse valvar irregularities or valvar thickening were not regarded as a vegetation..$^{13}$

\section{Echocardiographic criteria of abscess and} infectious myocardial infiltration

A perivalvar abscess was suspected if a region of reduced echodensity or echolucent cavities adjacent to the valves or within the valvar annulus was present. ${ }^{14}{ }^{15} \mathrm{~A}$ region of reduced echodensity inside the left ventricular myocardium was regarded as suspicious for infectious myocardial infiltration if it was distinct in echocardiographic appearance, detected throughout the cardiac cycle, and its diameter was larger than $0.5 \mathrm{~cm}$. Echolucent regions with a diameter smaller than $0.5 \mathrm{~cm}$ could not be differentiated from the heterogeneous echogenicity of a normal left ventricular myocardium and therefore were not regarded as suspicious for infectious myocardial infiltration.

\section{CLINICAL VARIABLES}

The following clinical events were recorded: (1) mortality; (2) cerebral or peripheral embolic events; (3) congestive heart failure occurring during acute infective endocarditis, documented by clinical findings and haemodynamic measurements; (4) cardiac valve surgery; (5) duration of fever and increase in plasma concentrations of acute phase reactants after the initiation of antibiotic treatment; (6) occurrence of gastrointestinal lesions in $S$ bovis endocarditis as defined by clinical symptoms in association with gastroscopic, colonoscopic, or ultrasonographic findings.

\section{MORPHOLOGICAL VARIABLES}

The presence, size, localisation, mobility, and consistency of valvar vegetations were evaluated according to transoesophageal echocardiographic findings. Mitral and tricuspid regurgitation was graded as mild, moderate, or severe using colour coded Doppler imaging, as described previously. ${ }^{20-23}$ Aortic regurgitation was graded by comparing the regurgitant jet width with the left ventricular outflow width. ${ }^{24}$ A valve was considered to be affected by infective endocarditis if a vegetation was visualised or if new or increased valve regurgitation was detected during the disease.

If echocardiography suggested a perivalvar abscess or infectious myocardial infiltration, the surgeon or the pathologist was informed. In 88 patients the presence of such lesions was evaluated on the basis of macroscopic and histological findings obtained by intraoperative biopsy or necropsy. A perivalvar abscess or infectious myocardial infiltration was considered to be present when extensive infection within the perivalvar tissue or the left ventricular myocardium was proven histologically.

\section{STATISTICAL ANALYSIS}

The occurrence of clinical and morphological variables in $S$ bovis and non- $S$ bovis endocarditis was compared using the Fisher's exact test. 


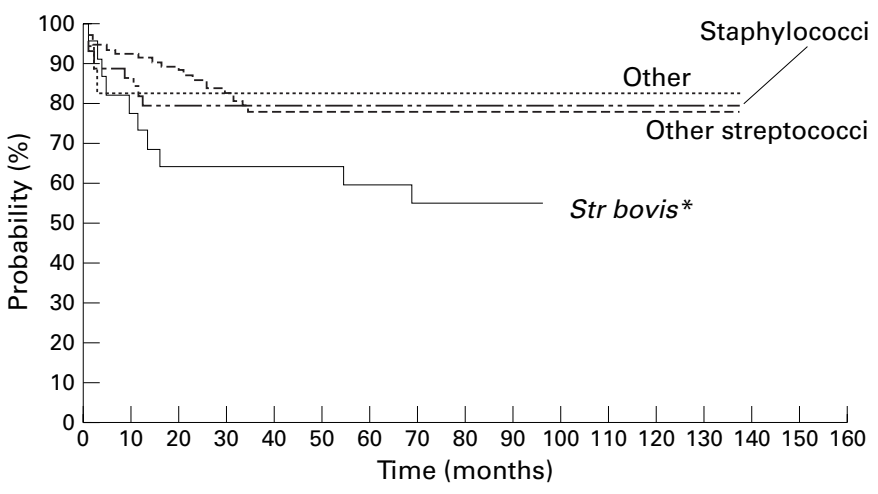

$\begin{array}{llllll} & \underline{6} & \underline{12} & \underline{24} & \underline{48} & \underline{96} \\ \text { Str bovis: } & 17 & 15 & 12 & 9 & 4 \\ \text { Other } & & & & & \\ \text { streptococci: } & 86 & 79 & 67 & 39 & 19 \\ \text { Staphylococci: } & 39 & 37 & 31 & 22 & 11 \\ \text { Other: } & 15 & 14 & 8 & 7 & 2\end{array}$

Figure 1 Cumulative survival rates in 177 patients with infective endocarditis between 1983 and 1996. (Numbers below graph are patients at risk 6, 12, 24, 48, and 96 months after hospital discharge.) ${ }^{\star} p<0.05$.

For continuous variables the Wilcoxon test was used. A probability ( $p$ ) value of $<0.05$ was considered to be significant. Mean values and the standard deviation were calculated for continuous variables. Patient survival curves were constructed according to the method of Kaplan and Meier. ${ }^{25}$ Survival curves were compared using the log-rank test.

\section{Results}

CLINICAL DATA

Table 1 presents clinical characteristics of the patients according to the causative microorganisms. In 10 of 22 patients (45\%) with $S$ bovis endocarditis, 16 gastrointestinal lesions were diagnosed (two gastric ulcers, one gastric cancer, two duodenal ulcers, two colon cancers, four colon adenomas, one inflammatory bowel disease, two colonic diverticula, one angiodysplasia, one liver cirrhosis). In $S$ bovis endocarditis the mean (SD) duration of fever and increased acute phase reactants after the onset of treatment was 7 (4) and 16 (6) days, respectively. When infective endocarditis was caused by other microorganisms, this period was 5 (2) $(\mathrm{p}=0.004)$ and 9 (4) days $(\mathrm{p}=0.001)$, respectively. Mortality was $45 \%$ (10 of 22 patients) and $25 \%$ (38 of 155 patients) in $S$ bovis and non-S bovis endocarditis, respectively

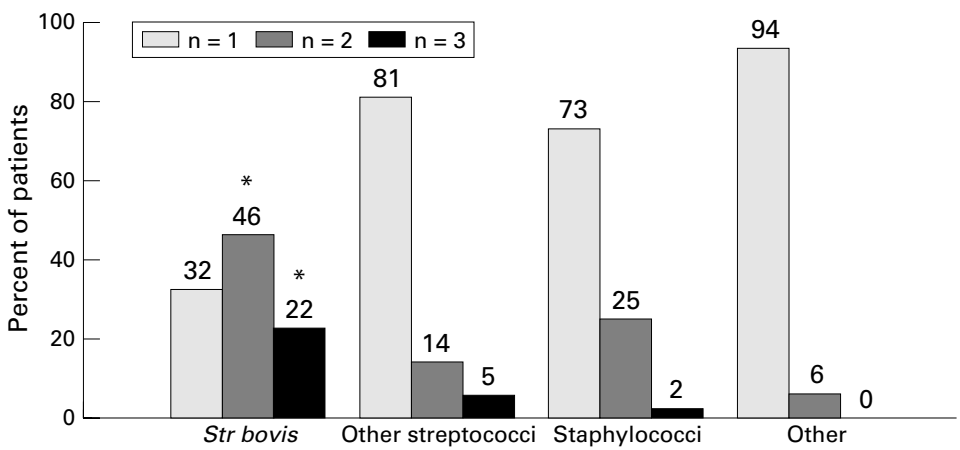

Figure 2 Frequency of multiple valve endocarditis in 177 patients. n, number of affected valves/patient.
Table 2 Causes of death in 10 patients with Streptococcus bovis endocarditis

\begin{tabular}{llll}
\hline Patient & $\begin{array}{l}\text { Age (years) } \\
\text { sex }\end{array}$ & $\begin{array}{l}\text { Follow up } \\
\text { (months) }\end{array}$ & Cause of death \\
\hline 5 & $59 / \mathrm{M}$ & 1 & Postoperative sepsis \\
26 & $70 / \mathrm{F}$ & 57 & Gastric cancer \\
44 & $68 / \mathrm{M}$ & 12 & Bowel carcinoma \\
51 & $72 / \mathrm{M}$ & 17 & Heart failure \\
68 & $76 / \mathrm{M}$ & 4 & Multiorgan failure \\
86 & $71 / \mathrm{F}$ & 10 & Heart failure \\
94 & $66 / \mathrm{M}$ & 14 & Heart failure \\
109 & $67 / \mathrm{F}$ & 72 & Liver cirrhosis \\
121 & $75 / \mathrm{M}$ & 5 & Postoperative sepsis \\
140 & $74 / \mathrm{F}$ & 3 & Postoperative sepsis \\
\hline Follow up, time between hospital referral and death.
\end{tabular}

$(\mathrm{p}=0.04)$. Patient survival curves are given in fig 1 . Of the patients with $S$ bovis endocarditis, $27 \%$ died for reasons directly related to the endocarditis, $18 \%$ from other underlying diseases or from diseases acquired during their hospital admission (table 2). Embolic events occurred in $9 \%$ (two of 22 patients) and $32 \%$ (50 of 155 patients) in $S$ bovis and non- $S$ bovis endocarditis, respectively $(\mathrm{p}=0.02)$. Congestive heart failure (New York Heart Association grade III or IV) occurred in 32\% (seven of 22 patients) and $24 \%$ (37 of 155 patients) in $S$ bovis and non-S bovis endocarditis, respectively (NS).

MORPHOLOGICAL DATA

In $S$ bovis endocarditis the simultaneous involvement of two or three valves was a common finding, in contrast to endocarditis caused by other microorganisms (fig 2). The mean (SD) number of vegetations per valve was 1.8 (0.7) in $S$ bovis endocarditis, versus $1.3(0.5)$ in the other groups $(p=0.05)$. The maximum vegetation length was less than $1.0 \mathrm{~cm}$ in $64 \%$ and $49 \%$ of the vegetations in $S$ bovis endocarditis and non- $S$ bovis endocarditis, respectively $(\mathrm{p}=0.01)$. In $S$ bovis endocarditis, $68 \%$ and $55 \%$ of the vegetations appeared to be completely fixed at the valve with no independent motion, and of higher echodensity, respectively, versus $40 \%(\mathrm{p}=0.03)$ and $33 \%$ $(p=0.04)$ in infective endocarditis caused by other microorganisms. Valvar damage in $S$ bovis endocarditis resulted in a higher percentage of patients presenting with moderate or severe regurgitation than in endocarditis caused by other microorganisms ( $86 \% v 76 \% ; \mathrm{p}=0.05)$. Intraoperative biopsy and necropsy showed a significantly higher frequency of left ventricular infectious myocardial infiltrations in $S$ bovis endocarditis than in non- $S$ bovis endocarditis $(36 \% v 10 \% ; \mathrm{p}=0.002)$. The frequency of perivalvar abscesses was comparable for all microorganisms.

\section{Discussion}

MORTALITY

Streptococcus bovis is identified as the causative microorganism in a minor proportion of patients with infective endocarditis, but commonly occurs in elderly people. ${ }^{26}$ Mortality rates in $S$ bovis endocarditis in previous studies ranged from $7.5 \%$ to $38 \%{ }^{27}{ }^{28}$ In other series of elderly people with infective endocarditis that included different causative microorganisms, 
mortality varied from $25 \%$ to $45 \% . .^{129}{ }^{30}$ The data suggest that mortality is not only related to the virulence of $S$ bovis, but also to the more frequent occurrence of underlying extracardiac diseases from which patients died during follow up. Initial symptoms such as fever and a raised white cell count are commonly masked in the elderly. ${ }^{29}$ This results in a therapeutic delay in this group of patients, which may cause a higher mortality during $S$ bovis endocarditis. The more frequent occurrence of heart failure and the need for extensive surgical interventions in the majority of patients with $S$ bovis endocarditis also contribute to the higher mortality in these patients.

\section{EMBOLIC EVENTS}

There were significantly fewer embolic events in $S$ bovis endocarditis than in infective endocarditis caused by other microorganisms, as has been reported previously. ${ }^{31}$ Other studies mentioned a lower incidence of embolic events and a higher proportion of $S$ bovis endocarditis in the elderly but did not analyse the embolic frequency in $S$ bovis cases separately. ${ }^{29}{ }^{32}$ The low frequency of embolic events correlates with the transoesophageal echocardiographic appearance of the vegetations, which were strikingly smaller than in the patients with non- $S$ bovis endocarditis, typically fixed, without oscillating parts, and of high echodensity. Vegetations showing these morphological characteristics are unlikely to embolise..$^{33} 34$

VALVE DAMAGE AND TISSUE DESTRUCTION

The data of our study suggest that the simultaneous involvement of two or three valves is more common in $S$ bovis endocarditis than in other forms of endocarditis. In two studies multiple valve involvement was present in 36\% and $60 \%$ of the patients with $S$ bovis endocarditis, respectively. ${ }^{28}{ }^{35}$ The more frequent attachment of multiple vegetations at one valve indicates the remarkable degree of valve damage in $S$ bovis endocarditis. This explains the higher proportion of haemodynamically relevant valve regurgitation than in infective endocarditis caused by other microorganisms. As a result, deterioration of left ventricular function and heart failure are more common. Severe multiple valve regurgitation and heart failure result in the need for extensive surgical interventions. Heart failure and extensive surgery are known to be major predictors of fatal outcome in infective endocarditis. ${ }^{36}$

The frequent occurrence of infectious myocardial infiltration in this study correlates with the longer duration of fever and the increased acute phase reactants, even after the onset of antibiogram directed antibiotic treatment. It is also likely to have an influence on the deterioration of left ventricular function during the disease process. The greater frequency of valvar damage and infectious myocardial infiltration might be caused by the longer therapeutic delay in $S$ bovis endocarditis.

GASTROINTESTINAL LESIONS

In our study an association with gastrointestinal lesions was observed in nearly half the patients. In accordance with previous studies, no specific lesion could be identified. ${ }^{62737}$ This underlines the need to examine the complete gastrointestinal tract when $S$ bovis endocarditis is diagnosed. ${ }^{38}$ Robbins and Klein consider it necessary to do regular follow up colonoscopy, owing to the detection of malignant gastrointestinal tumours two years after infective endocarditis. ${ }^{39}$

LIMITATIONS

Although our study was designed prospectively, it has the disadvantages of observational studies on consecutive patients in a tertiary referral centre like our university hospital. The majority of the patients were referred to our institution from community hospitals. These patients might be a selected cohort with a more severe illness than the average population with infective endocarditis. Thus differences in mortality and morbidity in comparison with other series might to some extent be a result of referral bias.

The diagnosis of a perivalvar abscess or an infectious myocardial infiltration was based on histopathological results obtained from intraoperative biopsy or necropsy. Most of the areas which were inspected intraoperatively by the surgeon were suspicious of infective tissue destruction on the basis of preoperative transoesophageal echocardiography. This helped avoid false positive diagnoses. We should, however, remember that at present necropsy represents the only ideal standard for morphological analysis. Although transoesophageal echocardiography is regarded as the clinical gold standard, accuracy was poorer in the beginning of this series than in recent years. Therefore, a false negative diagnosis cannot definitely be excluded.

\section{CLINICAL IMPLICATIONS}

This study confirms that $S$ bovis endocarditis is a severe illness with a worse long term prognosis than infective endocarditis caused by other microorganisms. Clinicians should be aware of the more frequent occurrence of infectious myocardial infiltration. Repeat transoesophageal echocardiographic investigations for early detection of infectious tissue destruction seem to be indicated. Owing to the frequent finding of severe valvar regurgitation, surgical interventions should be considered before deterioration in left ventricular function occurs. Our data also confirm the need for complete upper and lower gastrointestinal tract examination in patients with $S$ bovis endocarditis.

1 Terpenning MS, Buggy BP, Kauffman CA. Infective endocarditis: clinical features in young and elderly patients. Am $\mathcal{F}$ Med 1987;83:626-34

2 Verheul HA, Vanderbrink RBA, Vanreeland T, et al. Effects of changes in management of active infective endocarditis on outcome in a 25-year period. Am f Cardiol 1993;72: $682-7$.

3 Venezio FR, Westenfelder GO, Cook FV, et al. Infective endocarditis in a community hospital. Arch Intern Med 1982;142:789-92

4 Kaye D. Changing pattern of infective endocarditis. $A m \mathcal{F}$ Med 1985;78(suppl 6B):157-62.

5 Klein RS, Recco RA, Catalano MT, et al. Association of Klein RS, Recco RA, Catalano MT, et al. Association of
Streptococcus bovis with carcinoma of the colon. $N$ Engl $\mathcal{F}$ Med 1977;297:800-2.

6 Murray HW, Roberts RB. Streptococcus bovis bacteremia and underlying gastrointestinal disease. Arch Intern Med 1978;138:1079-99. 
7 Hoen B, Briancon S, Delahaye F, et al. Tumors of the colon increase the risk of developing Streptococcus bovis endocarditis: case

8 Watanakunakorn C. Streptococcus bovis endocarditis associated with villous adenoma following colonoscopy. $\mathrm{Am}$ Heart f 1988;116:1115-6.

9 Steinberg D, Naggar CZ. Streptococcus bovis endocarditis with carcinoma of the colon [letter]. $N$ Engl f Med 1977;297:1354-5.

10 Noble CJ, Uttley AHC, Falk RH, et al. Streptococcus bovis endocarditis and colonic cancer [letter]. Lancet 1978;i:767.

11 Friis EV, Sorensen AS. Streptococcus bovis as the cause of combined endocarditis of the aortic, mitral and tricuspid valves. Ugeskr-Laegr 1995;157:7012-13.

12 Mittermayer H, Nesser HJ, Ohlinger W. Endocarditis due to a penicillin-tolerant streptococcus bovis: microbiological findings and echocardiographic follow-up. Infection 1983; 11:302-6.

13 Erbel R, Rohmann S, Drexler M, et al. Improved diagnostic value of echocardiography in patients with infective
endocarditis by transesophageal approach. A prospective endocarditis by transesophageal
study. Eur Heart $\mathcal{F}$ 1988;9:43-53.

14 Daniel WG, Mügge A, Randolph PM, et al. Improvement in the diagnosis of abscesses associated with endocarditis by transesophageal echocardiography. N Engl F Med 1991; 324:795-800

15 Daniel WG, Schröder E, Nonnast-Daniel B, et al. Conventional and transesophageal echocardiography in the diagnosis of infective endocarditis. Eur Heart $\mathcal{F}$ 1987;8(suppl J):287-92.

16 Shapiro SM, Young E, De Guzman S, et al. Transesophageal echocardiography in diagnosis of infective endocarditis. Chest 1994;105:377-82.

17 Job FP, Franke S, Lethen $\mathrm{H}$, et al. Incremental value of biplane and multiplane transesophageal echocardiography for the assessment of active infective endocarditis. $A m \mathcal{F}$ Cardiol 1995;75:1033-7.

18 Erbel R, Liu F, Ge J, et al. Identification of high-risk subgroups in infective endocarditis and the role of echocarsubgroups in infective endocarditis and the

19 Durack DT, Lukes AS, Bright DK. New criteria for diagnosis of infective endocarditis: utilization of specific echocardiographic findings. Am $\mathcal{F}$ Med 1994;96:200-9.

20 Helmcke F, Nanda NC, Hsiung MC, et al. Color Doppler assessment of mitral regurgitation with orthogonal planes. Circulation 1987;75:175-83.

21 Susuki Y, Kambara H, Kadota K, et al. Detection and evaluation of tricuspid regurgitation using a real-time, twodimensional, color-coded, Doppler flow imaging system: comparison with contrast two-dimensional echocardiography and right ventriculography. Am f Cardiol 1986;57:81115 .

22 Castello R, Lenzen P, Aguirre F, et al. Quantitation of mitral regurgitation by transesophageal echocardiography with Doppler color flow mapping: correlation with cardiac catheterization. 7 Am Coll Cardiol 1992;19:1516-21.
23 Omoto R, Kyo S, Matsumura M, et al. Evaluation of biplane color Doppler transesophageal echocardiography in 200 consecutive patients. Circulation 1992;85:1237-47.

24 Perry GJ, Helmcke F, Nanda NC, et al. Evaluation of aortic insufficiency by Doppler color flow mapping. $7 \mathrm{Am}$ Coll Cardiol 1987;9:952-9.

25 Kaplan EL, Meier P. Nonparametric estimation of incomplete observations. F Am Stat Assoc 1958;53:457-81.

26 Delahaye F, Goulet V, Lacassin F, et al. Characteristics of infective endocarditis in France in 1991. A 1-year survey. Eur Heart F 1995;16:394-401.

27 Ravreby WD, Bottone EJ, Keusch GT. Group D streptococcal bacteremia with emphasis on the incidence and presentation of infections due to Streptococcus bovis. $N$ Engl $\mathcal{F}$ Med 1973;289:1400-3.

28 Ballet M, Gevigney G, Garé JP, et al. Infective endocarditis due to Streptococcus bovis. A report of 53 cases. Eur Heart 7 1995;16:1975-80.

29 Werner GS, Schulz R, Fuchs JB. Infective endocarditis in the elderly in the era of transesophageal echocardiography: clinical features and prognosis compared with younger patients. Am f Med 1996;100:90-7.

30 Robbins N, DeMaria A, Miller MH. Infective endocarditis in the elderly. South Med F 1980;73:1335-8.

31 Mansur AJ, Grinberg M, da Luz PL, et al. The complications of infective endocarditis. A reappraisal in the 1980s. Arch Intern Med 1992;152:2428-32.

32 Steckelberg JM, Murphy JG, Ballard D, et al. Emboli in infective endocarditis: the prognostic value of echocardiography. Ann Intern Med 1991;114:635-40.

33 Sanfilippo AJ, Picard MH, Newell JB, et al. Echocardiographic assessment of patients with infectious endocarditis: prediction of risk for complications. 7 Am Coll Cardiol 1991;18:1191-9.

34 Mügge A, Daniel WG, Frank G, et al. Echocardiography in infectious endocarditis: reassessment of prognostic implications of vegetation size determined by the transthoracic and the transesophageal approach. 7 Am Coll Cardiol 1989; 14:631-8.

35 Oumeskour B, Roudaut R, Texier-Maugein J, et al. Incidence and severity of cardiac involvement in Streptococcus bovis septicemia. Report of 10 cases. Ann Cardiol Angeiol Paris 1990;39:507-11.

36 Karalis DG, Blumberg EA, Vilaro JF. Prognostic significance of valvular regurgitation in patients with infective endocarditis. Am f Med 1991;90:193-7.

37 Brooks RJ, Ravreby WD, Keusch G. More on Streptococcus bovis endocarditis and bowel carcinoma. $N$ Engl $\mathcal{F} \mathrm{Med}$ 1978;298:572.

38 Tornos MP, Permanyer G. Significance of Streptococcus bovis endocarditis [letter]. Ann Intern Med 1993;118:572.

39 Robbins N, Klein RS. Carcinoma of the colon 2 years after endocarditis due to Streptococcus bovis. Am $\mathcal{F}$ Gastroenterol 1983;78:162-3. 